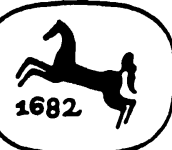

Sammlung Metzler Band 231 


\author{
Martin Lowsky
}

\title{
Karl May
}

J.B. Metzlersche Verlagsbuchhandlung Stuttgart 
CIP-Kurztitelaufnahme der Deutschen Bibliothek

\author{
Lowsky, Martin: \\ Karl May / Martin Lowsky. \\ - Stuttgart: Metzler, 1987. \\ (Sammlung Metzler; Bd. 231) \\ ISBN 978-3-476-10231-7 \\ ISBN 978-3-476-03934-7 (eBook) \\ DOI 10.1007/978-3-476-03934-7 \\ NE: GT
}

\title{
ISBN 978-3-476-10231-7 \\ ISSN 05583667
}

\section{231}

(c) 1987 Springer-Verlag GmbH Deutschland

Ursprünglich erschienen bei J. B. Metzlersche Verlagsbuchhandlung und Carl Ernst

Poeschel Verlag GmbH in Stuttgart 1987 


\section{Inhalt}

Vorwort............... XI

I. Materialien ................ 1

1. Der Nachlaß . . . . . . . . . . . . . . . . 1

2. Gesamtausgaben, wichtige Reihen . . . . . . . 4

3. Gesamtdarstellungen ........... . 7

4. Periodika, Bibliographien, Register . . . . . . . 10

II. Vita . . . . . . . . . . . . . . . . 13

1. Herkunft und frühe Kindheit (1842-1848) . . . . 16

2. Schul-, Seminar- und Lehrerzeit (1848-1863). . . . 18

3. Vagabondage und Haftzeit (1864-1874) . . . . . . 21

4. Anfänge als Schriftsteller (1874-1886). . . . . . . 24

5. Die Jahre des Erfolges (1887-1899) . . . . . . . 27

6. Die Altersperiode (1899-1912) . . . . . . . . 31

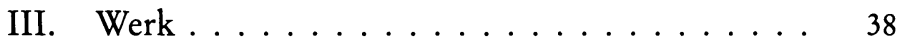

1. Das Frühwerk . . . . . . . . . . . . 38

2. Die Kolportageromane . . . . . . . . . . 43

3. Die klassischen Reise- und Jugenderzählungen . . . $\quad 52$

a) May als ethnographischer Autor . . . . . . . . . 57

b) Das Abenteuerliche . . . . . . . . . . . . . 62

In der Tradition des Abenteuerromans 62

Analytisches und aufklärerisches Erzählen 68

c) Einfache Formen . . . . . . . . . . . . 71

Einflüsse von Märchen und Sagen 71

Mays »Erlösungsmärchen « (Ueding) 76

d) Literaturpsychologische Sicht . . . . . . . . . . . 78

Kindheitliche Züge 78

Biographische Spiegelungen 80

Psychoanalytische Deutung: Mays »Recherche nach der verlorenen Liebe " (Wollschläger) 83

e) Die Reiseerzählungen als »ehrlicher Revolutionsersatz«(Bloch) . . . . . . . . . . 86 
Sozialkritische Beobachtungen 86 ,

Die Kolportage-Theorie Ernst Blochs 92

4. Die späten Reiseerzählungen . . . . . . . . . . 96

") Weihnacht! « 98

"Am Jenseits « 100

5. Das Alterswerk . . . . . . . . . . . 102

a) Die vier Altersromane: der »Großmystiker « May

(ArnoSchmidt) . . . . . . . . . . 106

»Im Reiche des silbernen Löwen «, Bd. 3, 4106

»Und Friede auf Erden! « 110

»Ardistan und Dschinnistan $« 114$

"Winnetou«, Bd. 4118

b) Autobiographische Schriften . . . . . . . . . . . 122

»Frau Pollmer, eine psychologische Studie « 122

"Mein Leben und Streben « 124

c) Novellen, Drama, Lyrik . . . . . . . . . . . . . . 128

IV. Rezeptions- und Forschungsgeschichte.

Ausblick . . . . . . . . . . . . . . . . . 132

Register .................. 146 


\section{Literatur}

Die folgenden Veröffentlichungen zitieren wir meist nur mit Verfasser und Jahreszahl.

Becker, Sibylle: Karl Mays Philosophie im Spätwerk. Ubstadt 1977 (MatKMF 3).

Bloch, Ernst: Über Märchen, Kolportage und Sage. In: Erbschaft dieser Zeit. Gesamtausgabe 4. Frankfurt a. M. 1962. S. 168-186.

Böbm, Viktor: Karl May und das Geheimnis seines Erfolges. Gütersloh ${ }^{2} 1979$.

Forst-Battaglia, Otto: Karl May. Traum eines Lebens - Leben eines Träumers. Bamberg 1966.

Hatzig, Hansotto: Karl May und Sascha Schneider. Bamberg 1967.

Jeglin, Rainer: Herrgottsengel, Rebell und Missionar. Anmerkungen zum Rettungsstil bei Karl May, SoKMG 24 (1980).

Kittler, Udo: Karl May auf der Couch? Ubstadt 1985 (MatKMF 9).

Klotz, Volker: Durch die Wüste und so weiter. Über Karl May. In: Akzente 9 (1962). S. 356-383. Auch in: Gerhard Schmidt-Henkel u.a. (Hg.): Trivialliteratur. Berlin 1964. S.33-51. (Auch in: Schmiedt, 1983, S. $75-100$.)

Klotz, Volker: Abenteuer-Romane. Sue - Dumas - Ferry - Retcliffe - May Verne. München/Wien 1979.

Maschke, Fritz: Karl May und Emma Pollmer. Bamberg 1973.

May, Karl: Mein Leben und Streben. Vorwort, Anmerkungen, Nachwort [...] von Hainer Plaul. Hildesheim/New York 1975.

Munzel, Friedhelm: Karl Mays Erfolgsroman \Das Waldröschen«. Hildesheim/New York 1979.

Oel-Willenborg, Gertrud: Von deutschen Helden. Weinheim/Basel 1973.

Roxin, Claus: Vorläufige Bemerkungen über die Straftaten Karl Mays. In: JbKMG 1971. S. 74-109.

Roxin, Claus: "Dr. Karl May, genannt Old Shatterhand«. Zum Bild Karl Mays in der Epoche seiner späten Reiseerzählungen. In: JbKMG 1974. S. 15-73.

Schmid, Euchar Albrecht [und Roland Schmid]: Gestalt und Idee. In: GW 34. ${ }^{29} 1975\left(-{ }^{37} 1985\right)$. S. 353-408.

Schmidt, Arno: Abu Kital. Vom neuen Großmystiker. In: Dya Na Sore. Karlsruhe 1958 (Reprint Frankfurt a. M. 1985). S.150-193. (Auch in: Schmiedt, 1983, S. 45-74). 
Schmidt, Arno: Sitara und der Weg dorthin. Karlsruhe 1963 (Reprint Frankfurt a. M. 1985).

Schmiedt, Helmut: Karl May. Studien zu Leben, Werk und Wirkung eines Erfolgsschriftstellers. Königstein/Ts. 1979.

Schmiedt, Helmut (Hg.): Karl May. Frankfurt a. M. 1983 (suhrkamp tb materialien).

Scholdt, Günter: Vom armen alten May. Bemerkungen zu "Winnetou IV« und der psychischen Verfassung seines Autors. In: JbKMG 1985. S. 102-151.

Stolte, Heinz: Der Volksschriftsteller Karl May. Radebeul 1936. Bamberg ${ }^{2} 1979$.

Ueding, Gert: Glanzvolles Elend. Versuch über Kitsch und Kolportage. Frankfurt a. M. 1973.

Ueding, Gert: Der Traum des Gefangenen. Geschichte und Geschichten im Werk Karl Mays. In: JbKMG 1978. S. 60-86. (Auch in: Scbmiedt, 1983, S. 160-187). Revid. u. erw. Fassung: Ueding, Gert: Auf fremden Pfaden in die Heimat. In: Die anderen Klassiker. Literarische Porträts aus zwei Jahrhunderten. München 1986. S. 156-183.

Wagner, Wolfgang: Der Eklektizismus in Karl Mays Spätwerk. SoKMG 16 (1979).

Wollschläger, Hans: „Die sogenannte Spaltung des menschlichen Innern, ein Bild der Menschheitsspaltung überhaupt «. Materialien zu einer Charakteranalyse Karl Mays. In: JbKMG 1972/73. S.11-92.

Wollschläger, Hans: Karl May. Grundriß eines gebrochenen Lebens. Zürich 1976. ${ }^{3} 1983$. 


\section{Abkürzungen}

DU Der Deutschunterricht

DVjs Deutsche Vierteljahrsschrift für Literaturwissenschaft und Geistesgeschichte

GAnz Graff-Anzeiger. Informationen über Reise- und Abenteuerschriftsteller

JbKMG Jahrbuch der Karl-May-Gesellschaft

KMJb Karl-May-Jahrbuch

LiLi Zeitschrift für Literaturwissenschaft und Linguistik

MatKMF Materialien zur Karl-May-Forschung

MfARU Magazin für Abenteuer-, Reise- und Unterhaltungsliteratur

M-KMG Mitteilungen der Karl-May-Gesellschaft

Repr Reprint

SoKMG Sonderheft der Karl-May-Gesellschaft

$\mathrm{Tb}$ Taschenbuch

Abkürzungen der Werkausgaben (s. S. Sf.):

DHSerie

EKMSerie

GelSerie

GesRE

GesRE (Repr)

GKSerie

GW

IllW

Olms

REE
Serie "Deutscher Hausschatz"

Serie »Erstdrucke Karl Mays in Faksimile-Ausgaben «

"Gelsenkirchener Serie“

Gesammelte Reiseerzählungen

Freiburger Erstausgaben (Reprint der Gesammelten Reiseerzählungen)

Serie "Der Gute Kamerad «

Gesammelte Werke

Illustrierte Werke

Olms Presse ("Waldröschen« u. a. Kolportageromane)

Reiseerzählungen in Einzelausgaben 


\section{Vorwort}

"Weißt du, Vater, an wen ich jetzt denke? « sagte sie. "An Karl May. Ich habe seine drei Bände sIm Lande des Mahdi< gelesen, und ---«

»Lies nicht das dumme Zeug von diesem May!« unterbrach er sie rasch und schnarrend. "Dieser Schriftsteller hat nichts als Phantasie [.]《

Diese Passage eines erlauschten Gesprächs steht bei Karl May, in seinem späten Roman »Und Friede auf Erden!« (1901/04), und man mag in ihr vielleicht ein anmaßendes Selbstbespiegeln des Autors, vielleicht aber auch eine moderne selbstreflektorische Haltung der Erzählerfigur erblicken. Allemal sind die Zeilen typisch für diesen Schriftsteller, der ungeniert wie kein anderer sein Wunsch-Ich in das Zentrum des Werkes stellte und nicht zuletzt hierdurch Lesermassen bezauberte und zugleich die Literaturwissenschaftler degoutierte. Seit etwa zwei Jahrzehnten jedoch hat sich um May eine weitgefächerte Forschung entwickelt, die, ursprünglich vom Einzug der Trivialliteratur in die Seminare beflügelt, sich längst in eigenen Kategorien bewegt und die früheren Versäumnisse der Germanistik fast vergessen macht.

Karl May hat, einem Balzac vergleichbar, mit Erbauungstexten und Dorfgeschichten debütiert und fünf endlose Kolportageromane verfaßt, bis er zu seinem Hauptwerk fand, zu den abenteuerlichen Reiseerzählungen, die mittlerweile ein Eigenleben als mythischer Kosmos angenommen haben und dieses noch in den banalsten Vermarktungen bewahren, und zu den pazifistischen Altersromanen, in denen das erzählende Ich in surrealistische Menschheitsutopien ausfährt. In Forschungsstand und -geschichte um diesen Autor, der nach Ernst Bloch »einer der besten deutschen Erzähler « ist, den Arno Schmit zum "Großmystiker « ernannte, der sich selbst im Alter als Psychologen bezeichnete und dessen Bücher ein Dreivierteljahrhundert nach seinem Tode jährlich in 2 Millionen Exemplaren verkauft werden, soll dieser Band der "Sammlung Metzler « einführen.

Für wertvolle Hilfe danke ich Ekkehard Bartsch (Bad Segeberg), Günter Baumann (Kiel), Walther Ilmer (Bonn) und ganz besonders Rolf Götz (Weilheim/Teck), Claus Roxin (Stockdorf) und meiner Frau Helga.

Kiel, im November 1986

Martin Lowsky 